\title{
Prevalence of Staphylococcus aurues carriage in patients with chronic kidney disease: A preliminary cross-sectional study of outpatients at the National Hospital of Sri Lanka
}

Nipun de Silva ( $\square$ nipunlak@gmail.com )

General Sir John Kotelawala Defence University https://orcid.org/0000-0002-4467-3659

M.N.F. Mufeena

National Hospital of Sri Lanka

Gayathri Sam

University of Colombo Faculty of Medicine

Shashika Dayarathna

University of Colombo Faculty of Medicine

Deepthi Kumari

University of Colombo Faculty of Medicine

Dilushi Wijayaratne

National Hospital of Sri Lanka

Geethika Patabendige

National Hospital of Sri Lanka

Saroj Jayasinghe

University of Colombo Faculty of Medicine

Research note

Keywords: Staphylococcus aureus, chronic kidney disease, carriage

Posted Date: July 16th, 2020

DOl: https://doi.org/10.21203/rs.3.rs-38875/v1

License: (c) (1) This work is licensed under a Creative Commons Attribution 4.0 International License.

Read Full License 


\section{Abstract \\ Objectives:}

To compare carriage rates of Staphylococcus aureus in patients with and without chronic kidney disease (CKD) and identify associated factors.

\section{Results:}

We enrolled 64 CKD $(A-1=13, A-2=17, A-3=34)$ and 56 non-CKD controls $(B)$. Patients were categorized as: stage 5 CKD on haemodialysis (A-1), stage 5 CKD not on haemodialysis (A-2), renal transplant recipients with glomerular filtration rate $>60 \mathrm{ml} / \mathrm{min}$ (A-3) and those having chronic medical diseases with glomerular filtration rate $>60 \mathrm{ml} / \mathrm{min} / \mathrm{m}^{2}(B)$. Median age $=57$ years (range $=17-77$ ). Nasal or axillary Staphylococcus aureus carriage was seen in 5 (38.5\%), 2 (11.8\%), 5 (14.7\%) and 9 (16.1\%) patients from $A-1$ to $A-3$ and $B$ groups respectively. All isolated organisms were methicillin sensitive. Haemodialysis $(p=0.035)$ and antibiotic use in the preceding month $(p=0.02)$ were associated with carriage. Demographic factors and co-morbidities were not associated with carriage.

\section{Introduction}

Staphylococcus aureus is an organism which commonly colonises the anterior nares and skin of humans. Generally $20 \%$ of healthy adults are persistent carriers, $30 \%$ intermittent carriers and $50 \%$ persistent non-carriers (1). These organisms usually live in harmony with their hosts, but become important pathogens when normal defenses are breached. Staphylococcus aureus is a common cause of blood stream infection and has a poor prognosis and mortality (2-4). Staphylococcal infections and skin carriage are commoner among dialysis patients, who are at risk of blood stream infection and death. Virulence factors that make $S$. aurues infections dangerous and challenging to treat include a microcapsule that interferes with opsonophagocytosis, toxin and enzyme production allowing tissue invasion, and the formation of biofilms on prosthetic devices and human tissues that are very difficult to eradicate with antibiotics (1).

Diabetes mellitus, prolonged haemodialysis-catheter use, and long-term dialysis are risk factors for Methicillin resistant Staphylococcus aureus (MRSA) carriage in chronic haemodialysis patients $(3,5)$. Pulmonary disease, recent hospital admission and antimicrobial use are additional risk factors (6).

Studies have failed to establish a causal relationship between haemodialysis and MRSA carriage, and higher carriage-rate could be nosocomial in origin (5). It has been suggested that regular screening and decolonization of MRSA-positive CKD carriers, healthcare workers and family members could reduce the risk of clinical infections (7). 
Rates of carriage in CKD vary between $2 \%-70 \%(3,5-7)$. We were unable to locate any studies that investigated the prevalence or risk factors associated with $S$. aureus colonizations among CKD patients in Sri Lanka.

The study proposed to determine the $S$. aureus carriage rates in different groups of patients with CKD compared to patients with non-renal chronic medical disorders, and to identify factors associated with $S$. aureus carrier state.

\section{Methods}

A cross sectional study was conducted at outpatient medical and nephrology clinics of the University Medical Unit of National Hospital of Sri Lanka from July to December 2017. The nephrology clinics included low-clearance, dialysis and post renal transplant clinics. All patients aged $>18$ years attending these clinics were considered eligible for the study, and consenting patients fulfilling the inclusion criteria of the categories listed below were recruited by consecutive sampling .

\section{A. Chronic kidney disease group}

Group A-1: Patients with End-stage kidney disease on regular haemodialysis

Group A-2: Patients with GFR $<15 \mathrm{ml} / \mathrm{min}$ (Sage $5 \mathrm{CKD}$ ) not receiving renal replacement therapy

Group A-3: Patients with a successful renal transplant and GFR $>60 \mathrm{ml} / \mathrm{min} / \mathrm{m}^{2}$

B. Controls

Patients with non-renal chronic medical illnesses and eGFR $>60 \mathrm{ml} / \mathrm{min} / \mathrm{m}^{2}$

Patients who have received treatment for $S$. aureus state within last six months were excluded from the study.

An interviewer administered pre tested data proforma with basic socio- demographic details and clinical details was used to collect information. Patients' clinical records were referred where relevant to collect additional information.

Thereafter swabs were taken for culture from anterior nares and skin (axillae, groin) by a trained medical officer. Transportation and processing of swabs was done according to standard operating procedures at microbiology laboratory, National Hospital of Sri Lanka. After collecting swabs they were placed in a freshly prepared Stuart's transport medium and closed. Swabs were transported immediately to the microbiology laboratory. Swabs were plated on agar, incubated at $32^{\circ} \mathrm{C}$, and grown for 24 hours. If $S$. aureus was grown testing for methicillin sensitivity was performed.

All data was entered into Statistical Package of Social Sciences (SPSS) 19 software. Prevalence of $S$. aureus carriage was calculated followed by analyzing the significance of associated factors. 
Dichotomous and numerical variables were analyzed using chi-square test and t-test respectively.

\section{Results}

We enrolled 64 CKD $(A-1=13, A-2=17, A-3=34)$ and 56 non-CKD controls. Median age $=57$ years (range $=17-77)$. Staphylococcus aureus carriage was seen in 5 (38.5\%), 2 (11.8\%), 5 (14.7\%) and 9 (16.1\%) patients from $A-1$ to $A-3$ and $B$ groups respectively. All were methicillin sensitive. Except an additional patient having skin carriage, all the other patients' skin and nasal carriage was similar. Summary of four study group participants and their carriage state is shown in Table 1.

Table 1

Characteristics and carriage rate of participants from each group

\begin{tabular}{|lllll|}
\hline Parameter & $\begin{array}{l}\text { A-1 }(\mathbf{n}= \\
\mathbf{1 3})\end{array}$ & $\begin{array}{l}\text { A-2 }(\mathbf{n}= \\
\mathbf{1 7})\end{array}$ & $\begin{array}{l}\text { A-3 }(\mathbf{3}= \\
\mathbf{3 4})\end{array}$ & $\mathbf{B}(\mathbf{n = 5 6})$ \\
\hline Female sex (percentage) & $6(46.2)$ & $5(29.4)$ & $11(32.4)$ & $38(67.9)$ \\
\hline Median age in years (Range) & $\begin{array}{l}50(28- \\
69)\end{array}$ & $\begin{array}{l}61(17- \\
76)\end{array}$ & $\begin{array}{l}60(21- \\
77)\end{array}$ & $\begin{array}{l}49.5(24- \\
68)\end{array}$ \\
\hline Nasal carriage (percentage) & $5(38.5)$ & $2(11.8)$ & $5(14.7)$ & $8(14.3)$ \\
\hline $\begin{array}{l}\text { Axillary and inguinal (skin) carriage } \\
\text { (percentage) }\end{array}$ & $5(38.5)$ & $2(11.8)$ & $5(14.7)$ & $9(16.1)$ \\
\hline
\end{tabular}

Chi-square test was performed to look for associated factors with carriage state. Haemodialysis $(p=$ $0.035)$ and antibiotic use during last month $(p=0.02)$ were associated with carriage. Sex, economic or education status, hospitalization within last year or diabetes mellitus were not associated with carriage. Findings are summarized in Table 2. 
Table 2

Association between Staphylocccus aurues carriage rate and risk factors

\begin{tabular}{|c|c|c|}
\hline Parameter & $\begin{array}{l}\text { Staphylococcus aureus colonization } \\
\text { rate }\end{array}$ & Significance \\
\hline Sex & 18.4 & 0.81 \\
\hline Female & 16.7 & \\
\hline \multicolumn{3}{|l|}{ Male } \\
\hline Age & 22.2 & 0.218 \\
\hline$>55$ years & 23.6 & \\
\hline \multicolumn{3}{|l|}{$<55$ years } \\
\hline Haemodialysis & 38.5 & 0.035 \\
\hline Yes & 15 & \\
\hline \multicolumn{3}{|l|}{ No } \\
\hline Antibiotic use during preceding month & 41.7 & 0.02 \\
\hline Yes & 14.8 & \\
\hline \multicolumn{3}{|l|}{ No } \\
\hline \multirow{2}{*}{$\begin{array}{l}\text { Hospital admission during preceding } \\
\text { month }\end{array}$} & 25 & 0.084 \\
\hline & 12.7 & \\
\hline \multicolumn{3}{|l|}{ No } \\
\hline Diabetes mellitus & 18.7 & 0.664 \\
\hline Yes & 15.6 & \\
\hline \multicolumn{3}{|l|}{ No } \\
\hline Hypertension & 12.7 & 0.053 \\
\hline Yes & 26.8 & \\
\hline \multicolumn{3}{|l|}{ No } \\
\hline Chronic liver cell disease & 11.1 & 0.6 \\
\hline Yes & 18 & \\
\hline No & & \\
\hline
\end{tabular}




\begin{tabular}{|llc|}
\hline Parameter & $\begin{array}{l}\text { Staphylococcus aureus colonization } \\
\text { rate }\end{array}$ & Significance \\
\hline Haemoglobin $<10 \mathrm{~g} / \mathrm{dL}$ & 27.6 & 0.1 \\
Yes & 14.3 & \\
No & & 0.748 \\
\hline Ischaemic heart disease & 15.4 & \\
Yes & 18.1 & \\
No & & \\
\hline
\end{tabular}

\section{Discussion}

This is the first reported study on Staphylococcus aureus carriage rate among patients with CKD compared to controls from Sri Lanka. Our key findings include high rates of $S$. aureus carriage among patients on haemodialysis and among patients who had received antibiotics recently. There was no methicillin resistance detected in our cohort and there was a good correlation between skin and nasal carriage.

Increased carriage among patients on haemodialysis noted in our study is comparable to reports from other countries (5). Hemodialysis patients are known to have poorer cell mediated immunity, antibody formation and phagocytic function and it has been suggested that such factors may increase the rates of colonization in this group. It would be interesting to observe the rates of colonization in patients with a similar degree of renal function who are on conservative management. In our cohort the median eGFR of the CKD 5 group was $13 \mathrm{ml} / \mathrm{min}$ and this would have been much higher than any residual renal function measured in the dialysis group. It is interesting that the rate of carriage in renal transplant patients with preserved graft function was $14.7 \%$ and was similar to that of controls. Most of our renal transplant recipients are on triple immunosuppression with prednisolone, a calcineurin inhibitor and an antiproliferative agent (azathiproine or mycophenolate mofetil) and the presence of good kidney function suggests that reasonable level of in-vivo immunsoppression had been achieved. This therefore indicates that there are factors beyond these arms of immunity that play a role in the high level of $S$. aureus carriage in the haemodialysis cohort. One explanation for the high rates of $S$. carriage in the haemodiaylsis cohorts is frequent exposure to health care settings and where cross contamination can occur with health care workers, and other patients. Further factors may be frequent venepuncture and the presence of prosthetic material. (10)

The concern regarding colonization in this population is the high risk of infection, particularly blood stream infection which has a high mortality. Up to $80 \%$ of the serotypes of Staphyloccocus causing blood stream infections are identical to isolates form the anterior nares and this suggests that most infection are auto-infections (1). We have no data on vascular access related infection rates in Sri Lanka, and this is an important area for research. Many of our patients present with advanced kidney disease, and are 
commenced on dialysis via temporary non-cuffed dialysis catheters that are kept in-situ for longer than advised by international recommendations. This is due to delays in access to theatre and radiological expertise for insertion of cuffed tunneled dialysis catheters and for creation of arteriovenous fistulae (AVF) or grafts (AVG). Therefore the bridging period between the initiation of haemodialysis and creation of an AVF is a high risk period for our patients. Our data was collected from patients who are already on haemodialysis and likely overestimates the prevalence of colonization in patients being initiated on haemodialysis. It would be interesting to prospectively follow up patients from the time of initiation of haemodialysis onwards, for colonization and infection rates within our cohort. Very few of our patients go on to receive long-term chronic dialysis, due to resource limitations, and therefore infection and colonization rates will be expected to differ from those from other settings. Eradication of $S$. aureus nasal colonization with topical mupriocin is known to reduce rates of Staphylococcus infection in haemodialysis patients (10). Information about our own infection rates will be useful for us to decide on the utility and cost-effectiveness of this approach in our setting.

Another interesting association we recognized was between antibiotic use during the preceding one month and Staphylococcus coonization. However, as this was a cross sectional study we could not conclude whether the skin carriage was the cause of more serious infection, subsequent to it or simply an association. There was no association between skin carriage and diabetes mellitus or recent hospitalizations in our study group (5). However there was a high rate of diabetes within our control group (54\%) and all the MSSA positive patients from the control group were diabetic.

None of our patients had MRSA colonization. This is in contrast to some of the cohorts showing high rates of colonization by both methicillin sensitive and resistant Staphylococcus aureus (8). Further studies are required to determine whether this depicts lower rates of antibiotic resistance in our setting or whether it is a result of selection bias from a single outpatient unit.

We noticed very good correlation between skin and nasal carriage in our samples. Only one had skin carriage without nasal carriage. This highlights the possible utility of testing for skin carriage or nasal carriage alone to detect a possible carrier status. Prior studies have confirmed this and shown that nasal swabbing alone is adequate $(8,9)$. This is in keeping with the results from our study. A few studies have higher detection rates using throat swabbing $(8,9)$. We did not adopt this due to inconvenience to the patient. It is also possible that we may have detected more carriage had we repeated screening as for intermittent carriage.

\section{Limitations}

Limitations of our study include a small sample size and the cross sectional nature of the study design. Strong associations could have been defined if a bigger sample from each group was included. It would have been interesting to follow up carriage and infection rates in a cohort of patients with CKD to observe how it varies after initiating dialysis and then post-transplant. 
We feel that the results from our preliminary study can be the basis for further large scale research on this interesting, yet neglected area in local setup. Recognising associations can enable identification of high risk patients who will benefit from screening and possibly decolonization.

\section{Abbreviations}

CKD- chronic kidney disease

GFR- Glomerular filtration rate

MRSA- Methicillin resistant Staphylococcus aureus

NHSL- National Hospital of Sri Lanka

\section{Declarations}

\section{Ethics approval and consent to participate}

Ethics clearance was obtained from Ethics Review Committee, National Hospital of Sri Lanka. Informed written informed consent was obtained from participants.

\section{Availability of data and material}

The datasets analysed during the current study are available from the corresponding author on reasonable request

\section{Consent for publication}

Not applicable

Competing interests

All authors declare that there are no competing interests

\section{Funding}

There was no specific funding involved with this study

\section{Author's contribution}


SJ conceived the study. NLdeS, GP and DW designed the study. MN, SG, SD and LK collected data. SG entered data. NLdeS and MN analysed data. NLdeS, SD and LK wrote the initial manuscript. SJ, SG and DW corrected the manuscript. All authors read and confirmed the final manuscript.

\section{Acknowledgements}

Authors would like to acknowledge staff of microbiology laboratory, National Hospital of Sri Lanka for the support provided.

\section{References}

1. Vandecasteele SJ, Boelaert JR, De Vriese AS. Staphylococcus aureus infections in hemodialysis: what a nephrologist should know. Clinical journal of the american society of nephrology. 2009;4(8):1388-400.

2. Lata C, Girard L, Parkins M, James MT. Catheter-related bloodstream infection in end-stage kidney disease: a Canadian narrative review. Canadian journal of kidney health disease. 2016;3(1):24.

3. Zimakoff J, Pedersen FB, Bergen L, Baagø-Nielsen J, Daldorph B, Espersen F, et al. Staphylococcus aureus carriage and infections among patients in four haemo-and peritoneal-dialysis centres in Denmark. J Hosp Infect. 1996;33(4):289-300.

4. Kim YS, Kim J. Higher Risk for All-cause Mortality of Staphylococcus aureus Bacteremia in Patients with Non-Dialysis Dependent Chronic Kidney Disease. Infection chemotherapy. 2020;52(1):82-92.

5. Yeoh LY, Tan FLG, Willis GC, Ooi ST. Methicillin-resistant Staphylococcus aureus carriage in hospitalized chronic hemodialysis patients and its predisposing factors. Hemodial Int. 2014;18(1):142-7.

6. Lu P-L, Tsai J-C, Chiu Y-W, Chang F-Y, Chen Y-W, Hsiao C-F, et al. Methicillin-resistant Staphylococcus aureus carriage, infection and transmission in dialysis patients, healthcare workers and their family members. Nephrology Dialysis Transplantation. 2008;23(5):1659-65.

7. Çelik G, Gülcan A, Dikici N, Gülcan E. Prevalence of nasal Staphylococcus aureus carriage in the patients undergoing hemodialysis and evaluation of risk factors and laboratory parameters. Ren Fail. 2011;33(5):494-8.

8. Bignardi G, Lowes S. MRSA screening: throat swabs are better than nose swabs. J Hosp Infect. 2009;71(4):373-4.

9. Young B, Votintseva A, Foster D, Godwin H, Miller R, Anson L, et al. Multi-site and nasal swabbing for carriage of Staphylococcus aureus: what does a single nose swab predict? J Hosp Infect. 2017;96(3):232-7. 